\title{
Numerical Analysis of Wind Turbine Airfoil Aerodynamic Performance with Leading Edge Bump
}

\author{
Majid Asli, Behnam Mashhadi Gholamali, and Abolghasem Mesgarpour Tousi
}

Center of Excellence on Computational Aerospace Engineering, Aerospace Engineering Department, Amirkabir University of Technology, Tehran, Iran

Correspondence should be addressed to Majid Asli; m_asli@aut.ac.ir

Received 16 May 2014; Revised 4 August 2014; Accepted 7 August 2014

Academic Editor: Roman Lewandowski

Copyright (c) 2015 Majid Asli et al. This is an open access article distributed under the Creative Commons Attribution License, which permits unrestricted use, distribution, and reproduction in any medium, provided the original work is properly cited.

Aerodynamic performance improvement of wind turbine blade is the key process to improve wind turbine performance in electricity generated and energy conversion in renewable energy sources concept. The flow behavior on wind turbine blades profile and the relevant phenomena like stall can be improved by some modifications. In the present paper, Humpback Whales flippers leading edge protuberances model as a novel passive stall control method was investigated on S809 as a thick airfoil. The airfoil was numerically analyzed by CFD method in Reynolds number of $10^{6}$ and aerodynamic coefficients in static angle of attacks were validated with the experimental data reported by Somers in NREL. Therefore, computational results for modified airfoil with sinusoidal wavy leading edge were presented. The results revealed that, at low angles of attacks before the stall region, lift coefficient decreases slightly rather than baseline model. However, the modified airfoil has a smooth stall trend while baseline airfoil lift coefficient decreases sharply due to the separation which occurred on suction side. According to the flow physics over the airfoils, leading edge bumps act as vortex generator so vortices containing high level of momentum make the flow remain attached to the surface of the airfoil at high angle of attack and prevent it from having a deep stall.

\section{Introduction}

Sustainable energy resources are becoming an increasing source of energy and developed rapidly. The research into clean and renewable energy resources, such as solar energy and wind power energy, is key subject of alternative energy development. The advantage of wind power systems is that such systems can generate electricity using clean and renewable energy without pollution. Moreover, wind power systems have minimum influence on the surrounding environment. Aerodynamic characteristics of wind turbine blades as the device to convert wind energy to electricity have the most important effect to produce energy efficiently. It is known that approximately $60 \%$ of the total energy loss of typical wind turbine systems is the aerodynamic loss. Since large horizontal-axis wind turbines are very expensive and operate for many years after the initial installation, it is important to design the wind turbine rotor blades in an aerodynamically efficient manner such that the maximum possible energy conversion can be achieved for the initial investment cost [1].
S809 airfoil is a common thick airfoil type for horizontalaxis wind turbine application which was developed by National Renewable Laboratory (NREL) to produce a low profile drag and maximum lift. Some static and dynamic experimental tests were carried out on this type of airfoil. Somers [2] designed and analysed S809 for horizontal-axis wind turbine application experimentally. Other experimental tests [3-7] were done which were very close to each other in static results at angles of attack before stall. Sheng et al. [8] experimentally tested the airfoil and explored its aerodynamic characteristics. The research showed that the airfoil has a complicated behaviour in dynamic stall condition. Xie et al. [9] investigate aerodynamic behaviour of the airfoil with slot and concluded that cascade with slots can increase the separation area with the increase of the angle of attack and also lift and drag coefficients increase and decrease, respectively, when angle of attack is 15 degrees and larger.

Active and passive flow control over the wing and airfoils for aerodynamic performance improvement have taken advantage of several techniques. Adding directly momentum 
to the boundary layer by jet flow techniques is as active stall control and modifying airfoil or wing shapes by using step or protuberance is as passive stall control mechanisms. Stall control is used to increase or to relocate the momentum in the flow direction within the boundary layer on an airfoil that generates flow separation in the flow direction [10]. Kang and Park [11] investigated an active stall control of wind turbine airfoil method using a continuous jet to improve the aerodynamic characteristics. The computed results showed a large flow control effect when the start position of the flow separation is the same as the slot position. All the stall control mechanisms try to keep boundary layer attached to the surface of the airfoil to prevent separation which leads to decreasing aerodynamic performance and energy conversion efficiency.

The concept of Humpback Whale pectoral flippers which have high level of manoeuvrability, shown in Figure 1, attracted researchers to investigate its aerodynamic characteristics in recent years. Pedro and Kobayashi [12] showed that the presence of stream wise vortices caused by protuberances improves aerodynamic performance of the wavy leading edge whale's similar airfoil. Johari et al. [13] investigated drag, lift, and pitching moments of NACA $63_{4}-021$ experimentally and showed that, for the airfoil with sinusoidal leading edge protuberances in angle of attacks higher than baseline airfoil stall, lift coefficient improves significantly with no penalty in drag coefficient. The research also revealed that amplitude of the protuberances has a distinct effect on the aerodynamic characteristics while wave length has little as van Nierop et al. [14] concluded the same results in hydrodynamic analysis analytically. In a separate work, Corsini et al. [15] explored cambered wavy leading edge airfoil versus symmetrical one numerically and concluded that lift curves feature an early recovery in poststall for symmetrical profile with an additional gain in lift for cambered airfoil specially applicable in fan and turbomachinery. Numerical analysis of the influence of passive leading edge tubercles on a lowaspect-ratio wing was performed by Watts and Fish [16]. This study showed $4.8 \%$ increase in lift, a $10.9 \%$ reduction in induced drag, and a $17.6 \%$ increase in lift-to-drag ratio at $10 \mathrm{deg}$. angle of attack on wings with tubercles over a baseline.

In the present paper, S809 as a thick type of airfoil which is applicable in wind turbine is considered to investigate its aerodynamic characteristics numerically. Navier-Stocks equations were solved in the discrete domain around the airfoil. Static performance investigation was compared to experimental results and therefore the effects of leading edge bump were considered in S809 during pre- and poststall regime of angles of attack.

\section{Airfoil Models}

S809 was employed as baseline airfoil and a C type mesh boundary was generated over the airfoil. A structured grid was selected because of controllability of mesh density over the airfoil and also increases in the performance of finite volume numerical method in comparison with unstructured mesh with hexahedral elements. The model has chord length

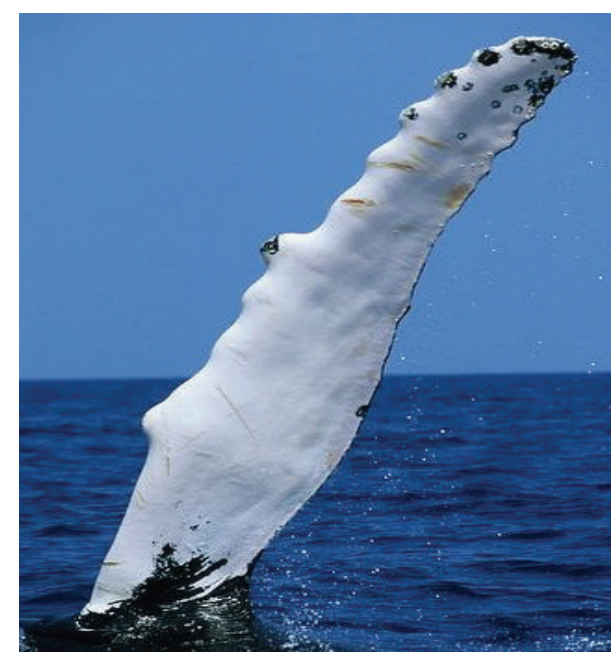

FIGURE 1: Humpback flipper which includes leading edge protuberances.

of $0.3 \mathrm{~m}$ and size of computational domain is $5.1 \times 3.6 \times 0.075$ which is considered as length $\times$ height $\times$ depth in SI unit. The inlet flow boundary is located at 6 chords upstream of the airfoil leading edge. The domain upper and lower boundaries are located at 6 chords and the flow outlet boundary is set at 10 chords away from the trailing edge of the airfoil to capture disturbance and flow in downstream area.

For the modified airfoil with leading edge protuberances, a sinusoidal wave was applied to the leading edge of the baseline airfoil. The wave length was set to $25 \%$ chord and amplitude of $2.5 \%$ of the baseline chord length is set to be self-consistent with the open literature as they were found in whales flipper. The domain size was set as the baseline model stated above.

The grid independency study was done to make sure of the optimum number of grids which lead to accurate results for solution convergence. If the grid is not generated fine enough, several important fluid phenomena could not be captured and the solution may not be converged. Three cases were considered with $600 \mathrm{k}, 1200 \mathrm{k}$, and $1700 \mathrm{k}$ cells. According to the results that were obtained, there are little differences in lift and drag coefficients between the last two cases. However, the difference between drag coefficient values of the first case and experimental data of Somers [2] was found considerable in the angles of attack higher than 10 degrees, since, in higher angles of attack, flow physics over the airfoil changes due to flow separation. So grids must be dense enough to capture flow vortices and physics. Therefore, the second case of $1200 \mathrm{k}$ cells was selected as the result of this grid independency study. Figure 2 shows the S809 airfoil model whose sinusoidal treatment was applied on its leading edge and the relevant computational domain and grids generated over the airfoil.

\section{Computational Modelling}

Here, numerical analysis was performed using threedimensional structured grids and solving the Navier-Stokes 


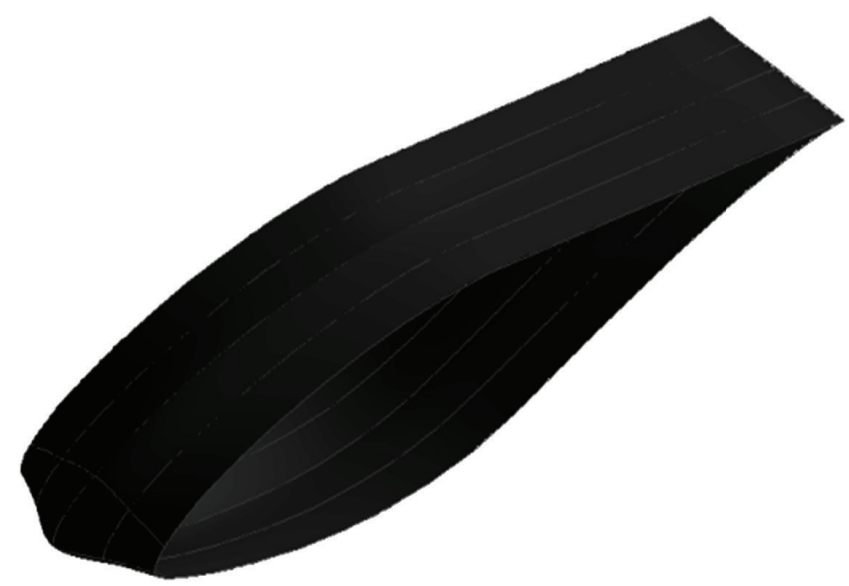

(a)

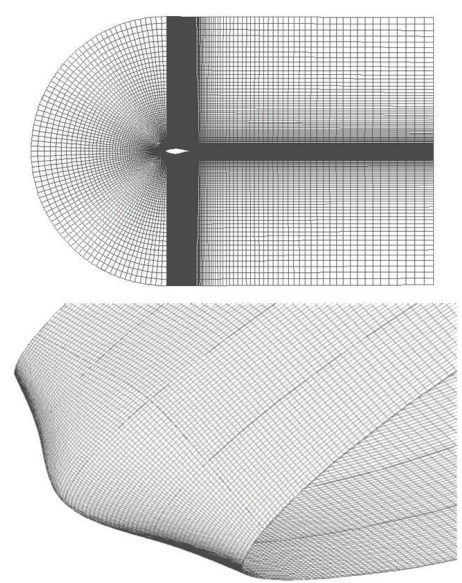

(b)

FIGURE 2: (a) Airfoil model with leading edge bump and (b) structured mesh over the airfoil.

equations, which comprise the conservation of mass, momentum, and energy as shown in the following equations, respectively:

$$
\begin{gathered}
\frac{\partial \rho}{\partial t}+\nabla \cdot(\rho U)=0 \\
\frac{\partial(\rho U)}{\partial t}+\nabla \cdot(\rho U \times U+p \overline{\bar{I}})=\nabla \cdot \overline{\bar{\tau}} \\
\frac{\partial(\rho U)}{\partial t}+\nabla \cdot\left[U\left(\rho e_{t}+p\right)\right]=\nabla \cdot(U \cdot \overline{\bar{\tau}})-\nabla \cdot Q .
\end{gathered}
$$

When pursuing further in the numerical solution of dynamic fluid flow using CFD techniques, in order to arrive at accurate solution, at least two aspects should be given careful attention, with due consideration of computational uncertainties. The first is the grid generation and the second is the turbulence modelling of the particular flow. The first is based on computational algorithm including the choice of appropriate grid; the grid fineness should be chosen to obtain efficient solution, which is a combination of computational speed and accuracy. The second is the turbulence modelling, which is based on physical modelling of real flow. Associated with turbulence modelling, the wall boundaries are the most common boundaries encountered in fluid flow problems [17].

Turbulent model of (SST) $k-\omega$ in the form of detached eddy simulation (DES) approach was applied for numerical simulation in the present paper. The hybrid model of DES attempts to combine the best aspects of RANS (Reynolds Averaged Navier-Stocks) and LES (large eddy simulation) methodologies in a single solution strategy where the LES mode is activated in regions where it is expected to have less accurate results than the RANS mode or the turbulent length scale goes over the grid dimension. This model takes advantage of the RANS model for near wall regions and treats the rest of the flow in LES manner.
The turbulent kinetic energy transport model equations of $k$ and $\omega$ can be stated by the following, respectively:

$$
\begin{aligned}
\frac{\partial(\rho k)}{\partial t}+\frac{\partial\left(\rho u_{j} k\right)}{\partial x_{j}}= & P-\beta^{*} \rho \omega k+\frac{\partial}{\partial x_{j}}\left[\left(\sigma_{k} \mu_{t}+\mu\right) \frac{\partial k}{\partial x_{j}}\right], \\
\frac{\partial \rho \omega}{\partial t}+\frac{\partial\left(\rho u_{j} \omega\right)}{\partial x_{j}}= & \frac{\gamma}{V_{t}} P-\rho \beta \omega^{2}+\frac{\partial}{\partial x_{j}}\left[\left(\sigma_{\omega} \mu_{t}+\mu\right) \frac{\partial \omega}{\partial x_{j}}\right] \\
& +2\left(1-F_{1}\right) \sigma_{\omega^{2}}\left(\frac{\rho}{\omega}\right)\left(\frac{\partial k}{\partial x_{j}}\right)\left(\frac{\partial \omega}{\partial x}\right),
\end{aligned}
$$

where

$$
\begin{gathered}
S_{i j}=0.5\left(\frac{\partial u_{j}}{\partial x_{i}}+\frac{\partial u_{i}}{\partial x_{j}}\right), \quad P=\tau_{i j} \frac{\partial u_{i}}{\partial x_{j}}, \\
\tau_{i j}=\mu_{t}\left(2 S_{i j}-\frac{2}{3} \frac{\partial u_{k}}{\partial x_{k}} \delta_{i j}\right)-\frac{2}{3} \partial k \delta_{i j} .
\end{gathered}
$$

Menter [18] developed the shear stress transport model by considering the $k-\omega$ formulation in the near wall region with the free stream independence of the $k-\omega$ model in the far field. Menter modified the dissipation term of turbulent kinetic energy which makes the SST $k$ - $\omega$ more reliable for adverse pressure gradient flows, and so forth. The dissipation kinetic energy is modified as the following equations:

$$
Y_{k}=\rho \beta^{*} k \omega f_{\beta^{*}},
$$

where

$$
\begin{gathered}
f_{\beta^{*}}=\max \left[\frac{L_{t}}{C_{\mathrm{des}} \Delta}, 1\right], \\
C_{\mathrm{des}}=0.61, \\
L_{t}=\frac{\sqrt{k}}{\beta^{*} \omega} .
\end{gathered}
$$




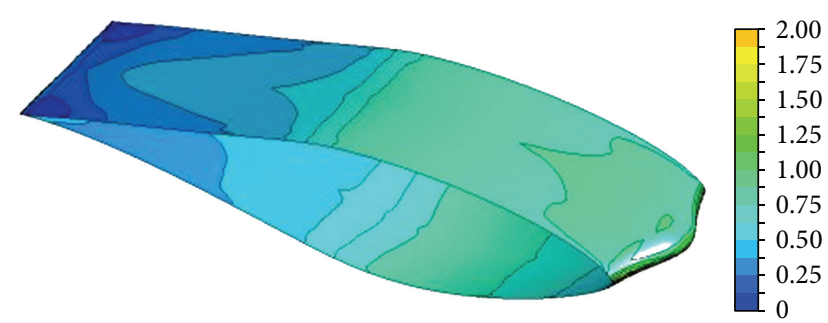

Figure 3: $y^{+}$distribution contour around the airfoil.

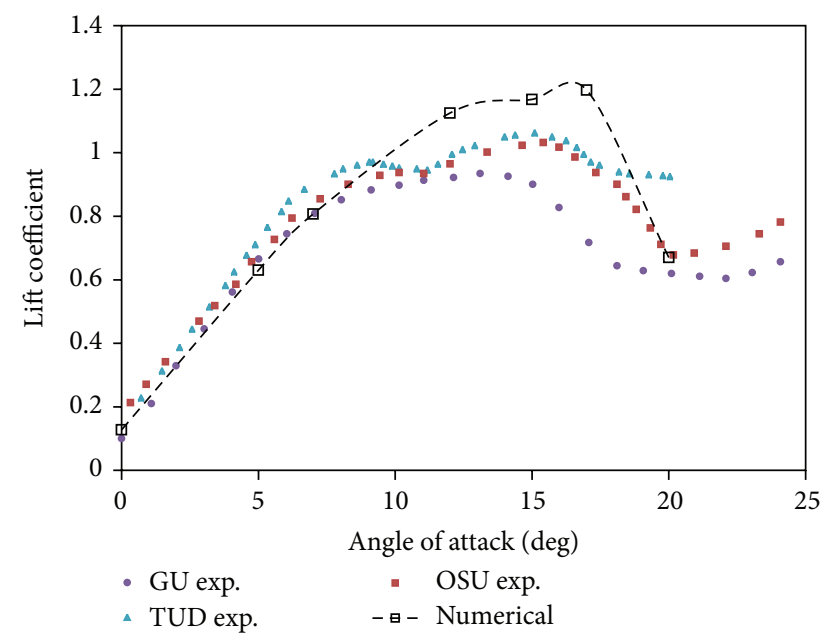

FIGURE 4: Lift coefficients of baseline airfoil obtained numerically versus experiments.

$L_{t}$ in the above relation is the turbulent length scale. Mahmoudnejad and Hoffmann $[19,20]$ validated the DES method in capturing all scales of the flow field.

The value of $Y$ plus $\left(y^{+}\right)$was checked over the airfoil to consider quality of the grids generated. $y^{+}$is a dimensionless number as a criterion to show whether the function in turbulent boundary layer is calculated correctly. Figure 3 shows the $y^{+}$distribution on the blade surface which is less than 2. This $y^{+}$shows a good accuracy of the wall treatment method in modelling the specifications of the boundary layer.

The models specifications are listed in Table 1.

\section{Results and Discussion}

The numerical results were compared to the experimental results of Somers [2], Sheng et al. [8], and Ramsay et al. [21] on S809 for validation, which were done at Delft University of Technology (TUD), Glasgow University (GU), and Ohio State University (OSU), respectively. Figure 4 shows the baseline lift coefficient obtained by numerical analysis versus the experiments. The numerical results show that the baseline airfoil stall starts around 17 degrees of AOA and lift coefficient decreases suddenly. The results of numerical solution have plausible agreement with the experiment, especially in prestall region. The maximum discrepancy occurred in stall start angle of attack, where separation occurred, which could be related to the impact of mesh distortion around the airfoil
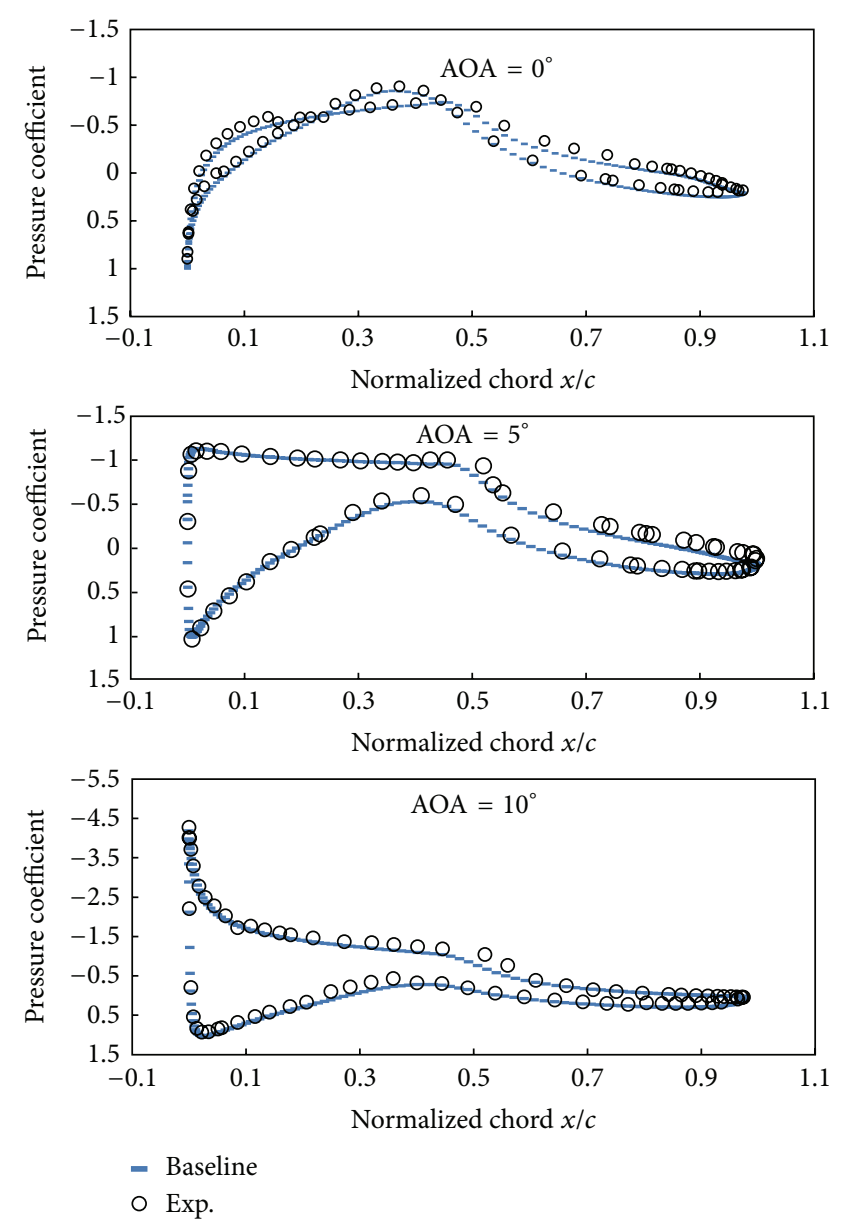

FIgURE 5: Pressure coefficient of baseline airfoil obtained numerically versus experiment [2].

TABLE 1: Model specifications for numerical analysis.

\begin{tabular}{lc}
\hline Airfoil types & $\begin{array}{c}\text { S809-straight L.E. } \\
\text { S809-sinusoidal L.E. }\end{array}$ \\
\hline Chord length $(c)$ & $0.3 \mathrm{~m}$ \\
Sinusoidal wave length & $25 \%$ of the chord \\
Sinusoidal amplitude & $2.5 \%$ of the chord \\
Reynolds number & $10^{6}$ \\
Turbulent model & DES-SST $k-\omega$ \\
Mesh size & $1200 \mathrm{k}$ \\
& $6 c$ upstream of the blade leading \\
Computational domain & edge \\
specification & $10 c$ downstream of the blade \\
& trailing edge \\
& $0.75 c$ in spanwise direction \\
\hline
\end{tabular}

and also the lateral blade interaction because of pitch-wise periodicity and numerical model as well. However in larger angle of attack around 20 degrees, the numerical results can predict the flow as experiments claimed. This verification of baseline airfoil is used as reference of numerical simulation of sinusoidal leading edge airfoil. Figure 5 indicates the pressure 


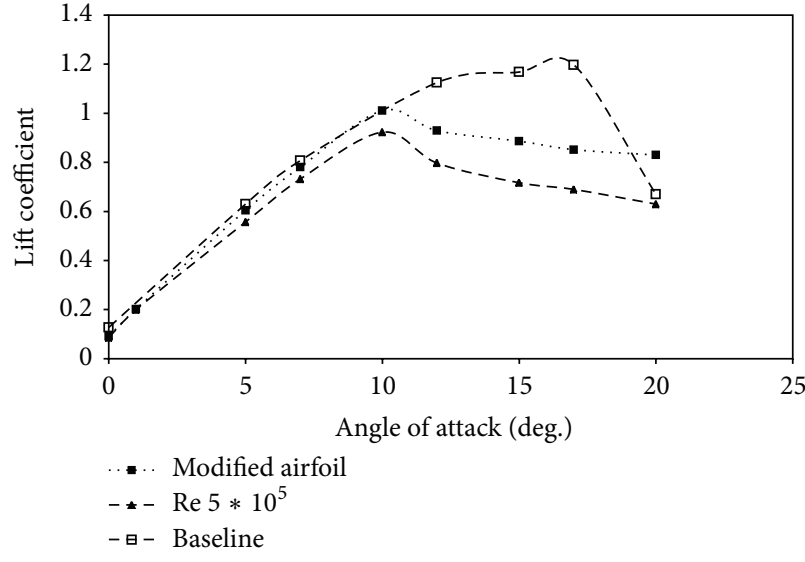

(a)

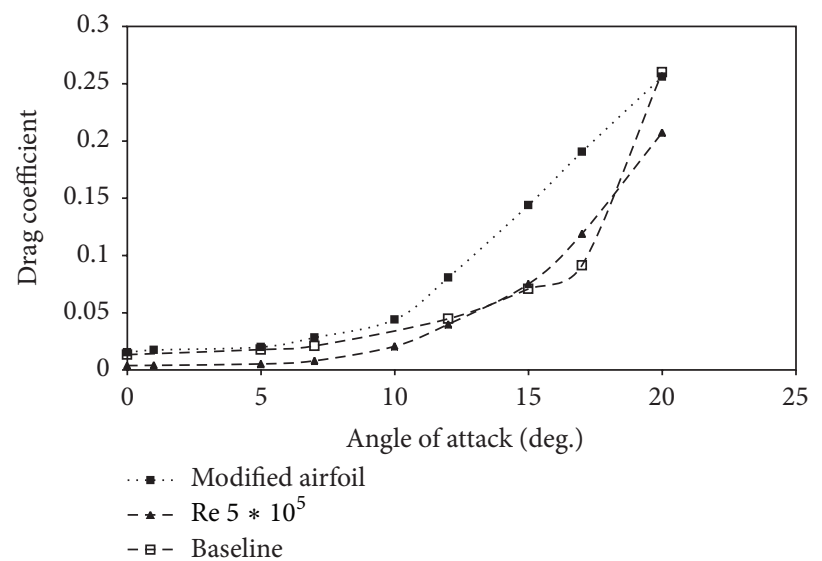

(b)

Figure 6: Comparison of lift (a) and drag (b) coefficients of the baseline airfoil with sinusoidal leading edge model.

coefficient distribution over the baseline airfoil obtained by Somers [2] and numerical solution in three angles of attack of 0,5 , and 10 degrees. This figure shows a good agreement between these results and indicates the accuracy of numerical simulation here.

The airfoil with sinusoidal leading edge was numerically investigated and compared to the baseline results in two Reynolds Numbers of $10^{6}, 5 \times 10^{5}$. Lift and drag coefficients are calculated and plotted in Figure 6. According to the lift coefficient obtained by the numerical analysis, the modified airfoil lift coefficient is not improved at low angle of attack. When separation of flow starts from the trailing edge of the airfoil, the difference between lift coefficient of the baseline airfoil and the modified model is increased. The modified airfoil stall occurred earlier than the baseline where there is no noticeable separation. A smooth stall and reduction of lift coefficient are observed for the modified airfoil. The lift coefficient results of the two airfoils meet each other at poststall regime while the flow is fully separated over the airfoil. On the other hand, drag coefficient in the two airfoils has the same trend. Drag coefficient of the modified airfoil is slightly larger than the baseline but it increases smoother than the baseline drag coefficient. Therefore it revealed that there is a little penalty in drag coefficient for the modified airfoil. Results for lower Reynolds number obtained numerically were plotted as lift and drag coefficients which confirm the trend of the modified airfoil behaviour with lower values.

To give additional insight into the flow behaviour, pressure contour and path lines of the flow over the modified model in three angles of attack of 5, 15, and 20 degree are indicated in Figure 7. According to the pressure contour of the modified airfoil plotted in $6 \pi$ spanwise direction of sinusoidal leading edge, it can be seen that flow over the modified airfoil has the period of $4 \pi$ in spanwise direction where all the phenomena are repeated periodically. In addition, according to the path lines, flow over the modified airfoil is completely attached at $\mathrm{AOA}=5 \mathrm{deg}$. However, in high angle of attack of $15 \mathrm{deg}$. streamlines were deviated and create vortices while the flow is separated from the suction side. Flow is diverged from pick of the bump and makes a swirl vortex in trough region. As angle of attack is increased, these vortices become greater and, at $\mathrm{AOA}=$ 20 deg., it can be seen that there are big vortices over the airfoil. These vortices make the flow more attached rather than completely separated in baseline model. The variation of vorticity in sinusoidal leading edge airfoil is much higher than the baseline. It can be seen that the presence of leading edge protuberances in the airfoil can act as vortex generators rather than baseline. These vortices carrying the high momentum flow delay the flow separation over the airfoil by reenergizing and adding the momentum to the boundary layer flow over the airfoil. Increase in drag coefficient because of vortices generated by leading edge bump is lower than increase in drag coefficient due to separation. So, the drag penalty of leading edge protuberances is preferable.

The pressure coefficient distribution on the airfoil with leading edge bump is indicated in Figure 8 in three sections of 1,2 , and 3 which related to the left trough, bump, and right trough of the modelled airfoil, respectively. Considering these figures, as angle of attack is increased, pressure gradient is increased at trailing edge which corresponds to flow separation there. As the flow deflects from sections 1 and 2 to section 3 , some vortices are generated in cross section 1 . Therefore, as angle of attack is increased, the vortices become larger in which at high angle of attack reach cross section 2. At cross section 3 less vortices exist rather than the other sections. These nonuniform behaviours make the airfoil have a smother aerodynamic characteristic in high angle of attacks.

\section{Conclusion and Further Work}

This paper presented three-dimensional numerical results obtained by simulation of flow over the S809 airfoil as a thick type of airfoil which is applicable in wind turbine. Firstly, three-dimensional infinite baseline airfoil was investigated numerically and compared to the recent experimental results for validation. Afterward, implementing the sinusoidal leading edge was considered. The impact of sinusoidal leading 

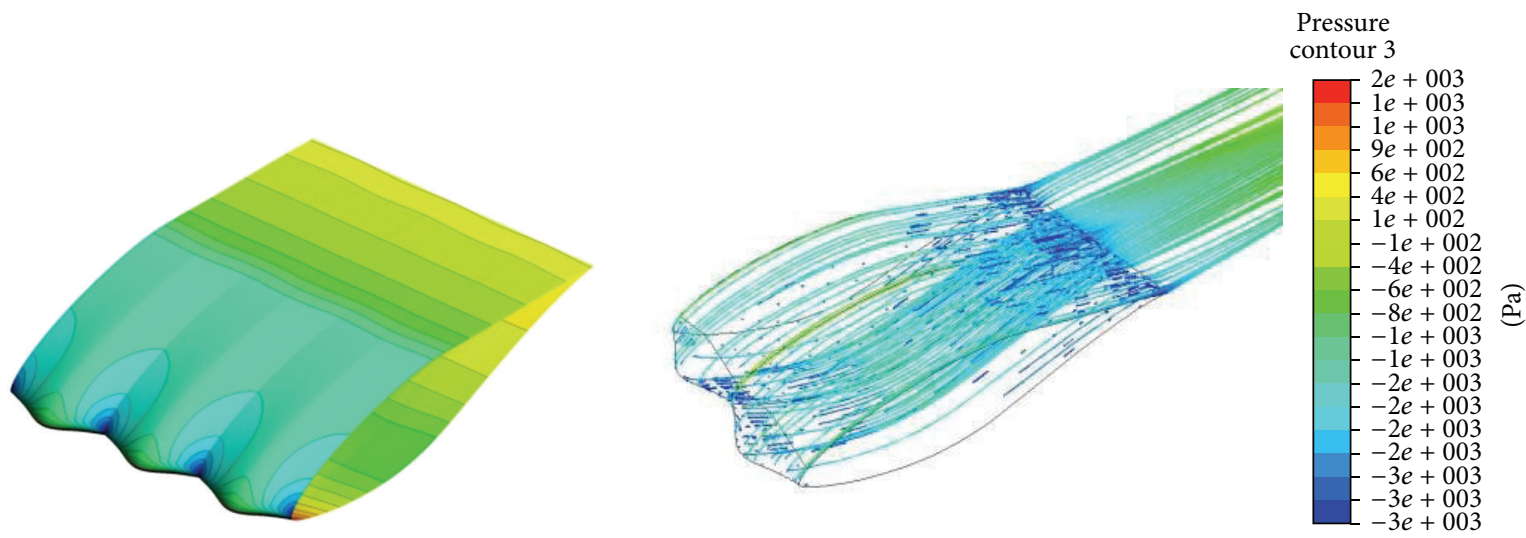

(a) $\mathrm{AOA}=5 \mathrm{deg}$.
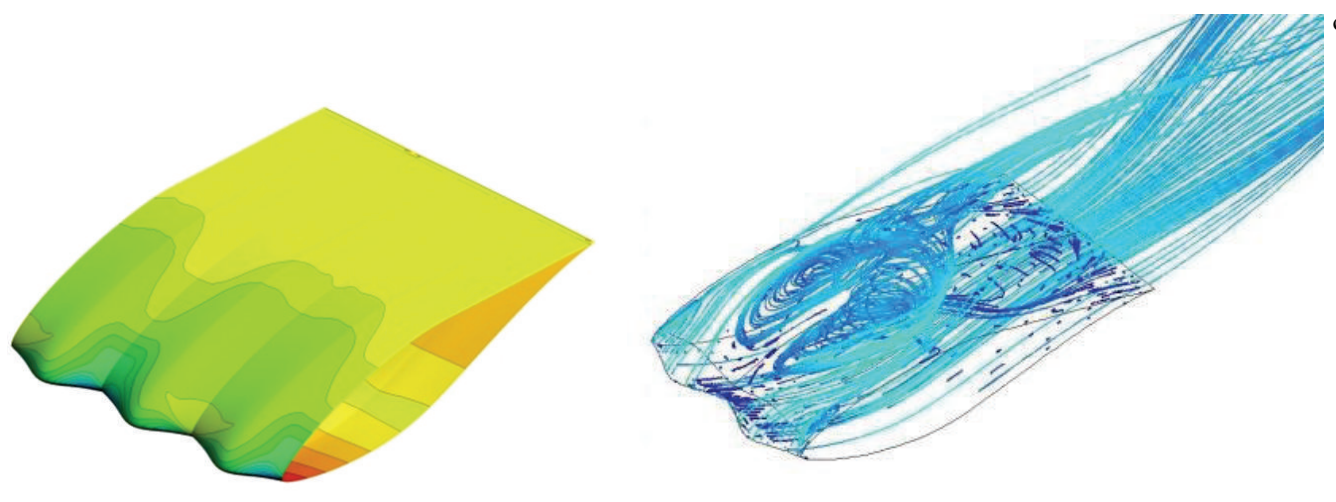

Pressure tour 3

(b) $\mathrm{AOA}=15 \mathrm{deg}$.
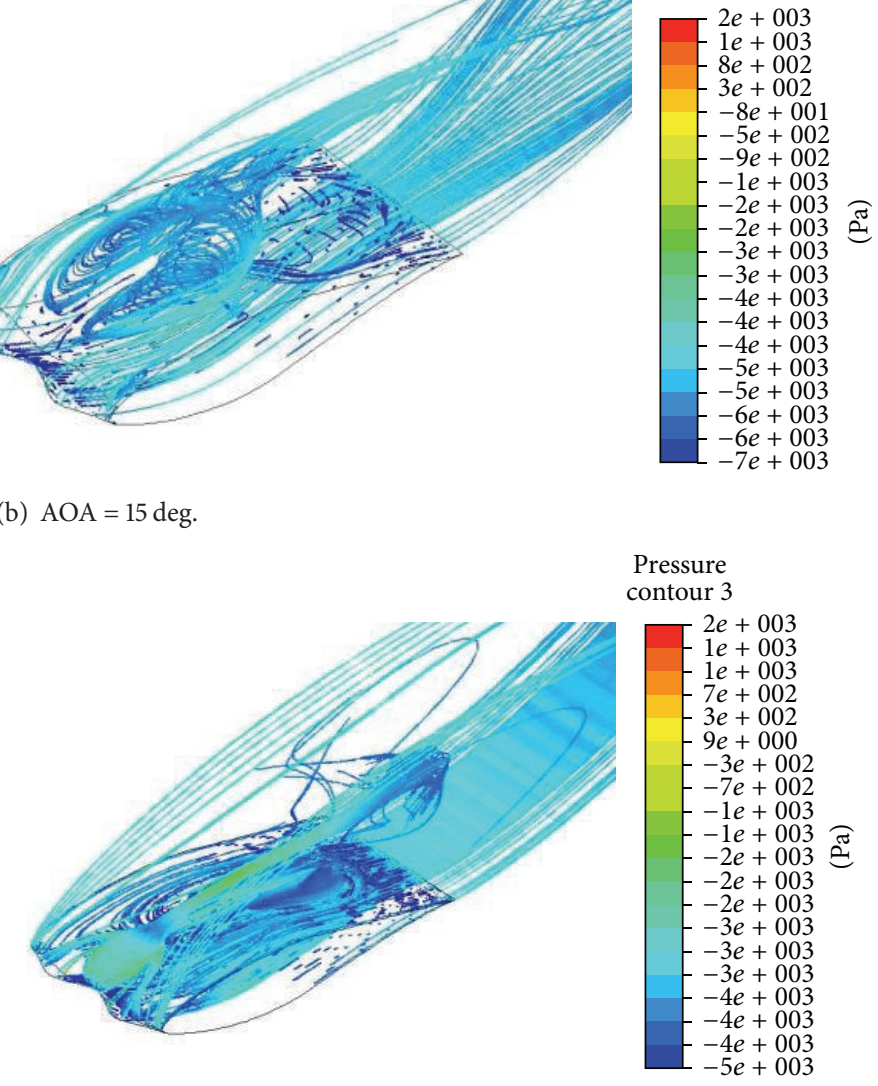

(c) $\mathrm{AOA}=20 \mathrm{deg}$.

Figure 7: Pressure contour at the left side and path line at the right side for modified airfoil at (a) AOA = 5 deg., (b) AOA = 15 deg., and (c) $\mathrm{AOA}=20 \mathrm{deg}$.

edge protuberances on aerodynamic characteristics of the airfoil as a new passive stall control method was investigated at different operating conditions ranging pre- to stall conditions. Wave length and amplitude of sinusoidal leading edge were chosen similar to Humpback Whale flippers.

The study revealed that flow physics over the airfoil has completely different behaviour in the leading edge bumpy airfoil compared to the baseline model. At low angle of attacks before stall inception region, flow over the bumpy leading edge airfoil contains vortices which decrease lift slightly rather than the baseline model due to forces induced by vortices. As the separation territory on the suction side of the airfoil in bumpy leading edge is larger than the baseline airfoil, the drag force is greater in all operating ranges studied here. When the flow separation starts to occur over the bumpy leading edge airfoil (AOA $\approx 12$ deg.), the lift decreases 

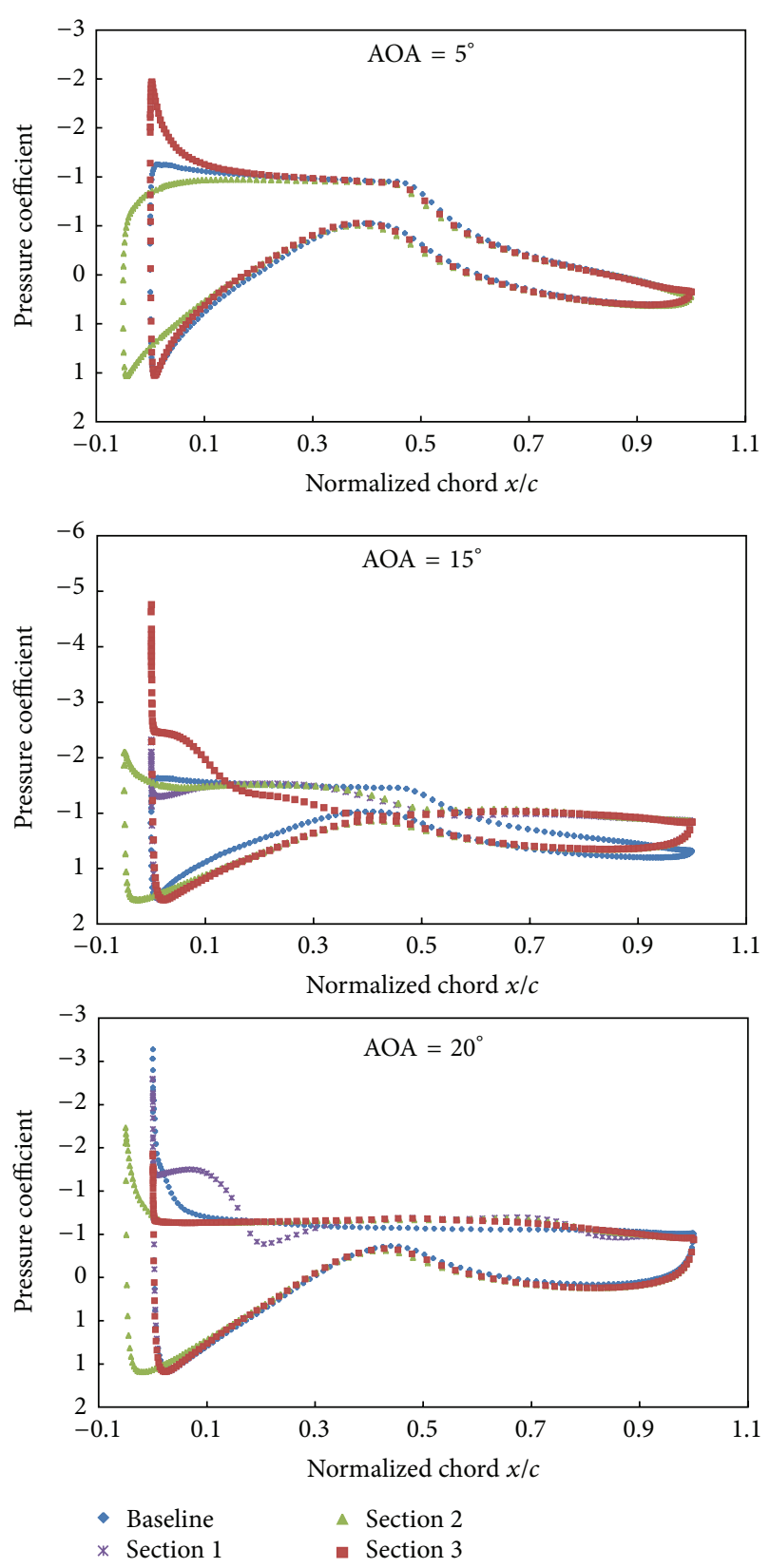

FIGURE 8: Chord-wise pressure coefficient distribution in three sections.

slightly and as angle of attack increases it is maintained in a range (about $\mathrm{Cl}=0.8$ ). This is mainly due to vortices generated by protuberances which contain relatively high level of momentum. This amount of momentum keeps the boundary layer attached to the surface and prevents large separation and sharp decrease in lift coefficient. According to the present study, it can be concluded that using leading edge protuberances on the thick airfoil can help increasing the attached flow region at high angles of attack with the little penalty of larger drag coefficient. It is valuable to do experimental research on applying protuberances on wind turbine blade geometry and investigate the flow characteristic over range of operating conditions with introducing an active mechanism to change the blade leading edge.

\section{Conflict of Interests}

The authors declare that there is no conflict of interests regarding the publication of this paper.

\section{References}

[1] H. I. Kwon, J. Y. You, and O. J. Kwon, "Enhancement of wind turbine aerodynamic performance by a numerical optimization technique," Journal of Mechanical Science and Technology, vol. 26, no. 2, pp. 455-462, 2012.

[2] D. M. Somers, "Design and experimental results for the S809 airfoil,” NREL Report No. NREL/SR-400-6918, 1997.

[3] D. A. Simms, M. M. Hand, L. J. Fingersh, and D. W. Jager, "Unsteady aerodynamics experiment phases II-IV test configurations and available data campaigns," NREL Report NREL/TP500-29955, 1999.

[4] M. M. Hand, D. A. Simms, L. J. Fingersh et al., "Unsteady aerodynamics experiment phase VI: wind tunnel test confi gurations and available data campaigns," NREL Report No. NREL/TP-500-25950, 2001.

[5] W. Sheng, R. A. Galbraith, F. N. Coton, and R. Gilmour, "The collected data for tests on an S809 airfoil, volume I: pressure data from static, ramp and triangular wave tests," G.U. Aero Report 0606, University of Glasgow, 2006.

[6] W. Sheng, R. A. M. Galbraith, F. N. Coton, and R. Gilmour, "The collected data for tests on an S809 airfoil, volume II: pressure data from static and oscillatory tests," G.U. Aero Report 0607, University of Glasgow, 2006.

[7] W. Sheng, R. A. Galbraith McD, F. N. Coton, and R. Gilmour, "The collected data for tests on the sand stripped S809 airfoil, volume III: pressure data from static, ramp and oscillatory tests," G.U. Aero Report 0608, University of Glasgow, 2006.

[8] W. Sheng, R. A. M. Galbraith, and F. N. Coton, "On the S809 airfoil's unsteady aerodynamic characteristics," Wind Energy, vol. 12, no. 8, pp. 752-767, 2009.

[9] Y. Xie, J. Chen, H. Qu, G. Xie, D. Zhang, and M. Moshfeghi, "Numerical and experimental investigation on the flow separation control of S809 airfoil with slot," Mathematical Problems in Engineering, vol. 2013, Article ID 301748, 14 pages, 2013.

[10] M. Gad-el-Hak, Flow Control, Cambridge University Press, Cambridge, UK, 2007.

[11] T. Kang and W. Park, "Numerical investigation of active control for an S809Wind turbine airfoil," International Journal of Precision Engineering and Manufacturing, vol. 14, no. 6, pp. 1037-1041, 2013.

[12] H. T. C. Pedro and M. H. Kobayashi, "Numerical study of stall delay on humpback whale Flippers," in Proceedings of the 46th AIAA Aerospace Sciences Meeting and Exhibit, Reno, Nev, USA, January 2008.

[13] H. Johari, C. Henoch, D. Custodio, and A. Levshin, "Effects of leading-edge protuberances on airfoil performance," AIAA Journal, vol. 45, no. 11, pp. 2634-2642, 2007.

[14] E. A. van Nierop, S. Alben, and M. P. Brenner, "How bumps on whale flippers delay stall: an aerodynamic model," Physical Review Letters, vol. 100, no. 5, Article ID 054502, 2008. 
[15] A. Corsini, G. Delibra, and A. G. Sheard, "On the role of leadingedgebumps in the control of stall onset in axial fan blades," Journal of Fluid Engineering, vol. 135, pp. 1-9, 2013.

[16] P. Watts and F. E. Fish, "The influence of passive, leading edge tubercles on wing performance," in Proceedings of the 12th International Symposium on Unmanned Untethered Submersible Technology (UUST '01), Autonomous Undersea Systems Institute, Lee, NH, USA, August 2001.

[17] H. Djojodihardjo, M. F. Abdul Hamid, A. A. Jaafar et al., "Computational study on the aerodynamic performance of wind turbine airfoil fitted with coand jet," Journal of Renewable Energy, vol. 2013, Article ID 839319, 17 pages, 2013.

[18] F. R. Menter, "Two-equation eddy-viscosity turbulence models for engineering applications," AIAA Journal, vol. 32, no. 8, pp. 1598-1605, 1994.

[19] N. Mahmoudnejad and K. A. Hoffmann, "Prediction of flow dynamics over cavities by detached eddy simulation," Journal of Aircraft, vol. 47, no. 6, pp. 1991-2004, 2010.

[20] A. K. Malipeddi, N. Mahmoudnejad, and K. A. Hoffmann, "Numerical analysis of effects of leading-edge protuberances on aircraft wing performance," Journal of Aircraft, vol. 49, no. 5, pp. 1336-1344, 2012.

[21] R. R. Ramsay, M. J. Hoffmann, and G. M. Gregorek, "Effects of grit roughness and pitch oscillations on the S809 airfoil," NREL Report NREL/TP-442-7817, 1995. 


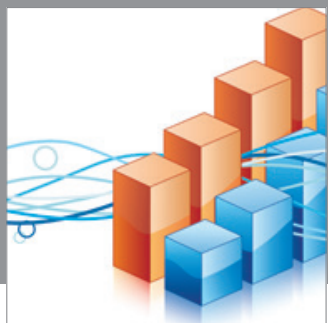

Advances in

Operations Research

mansans

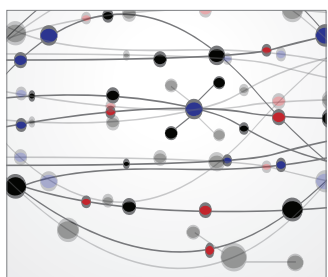

The Scientific World Journal
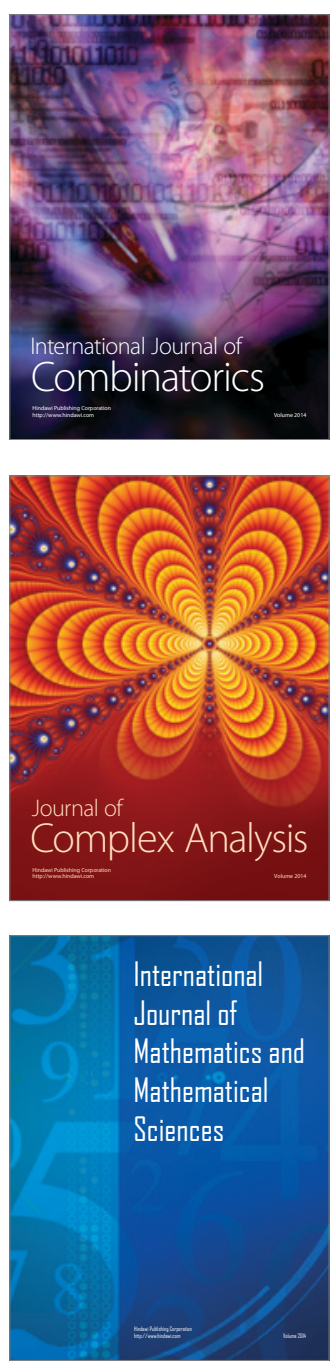
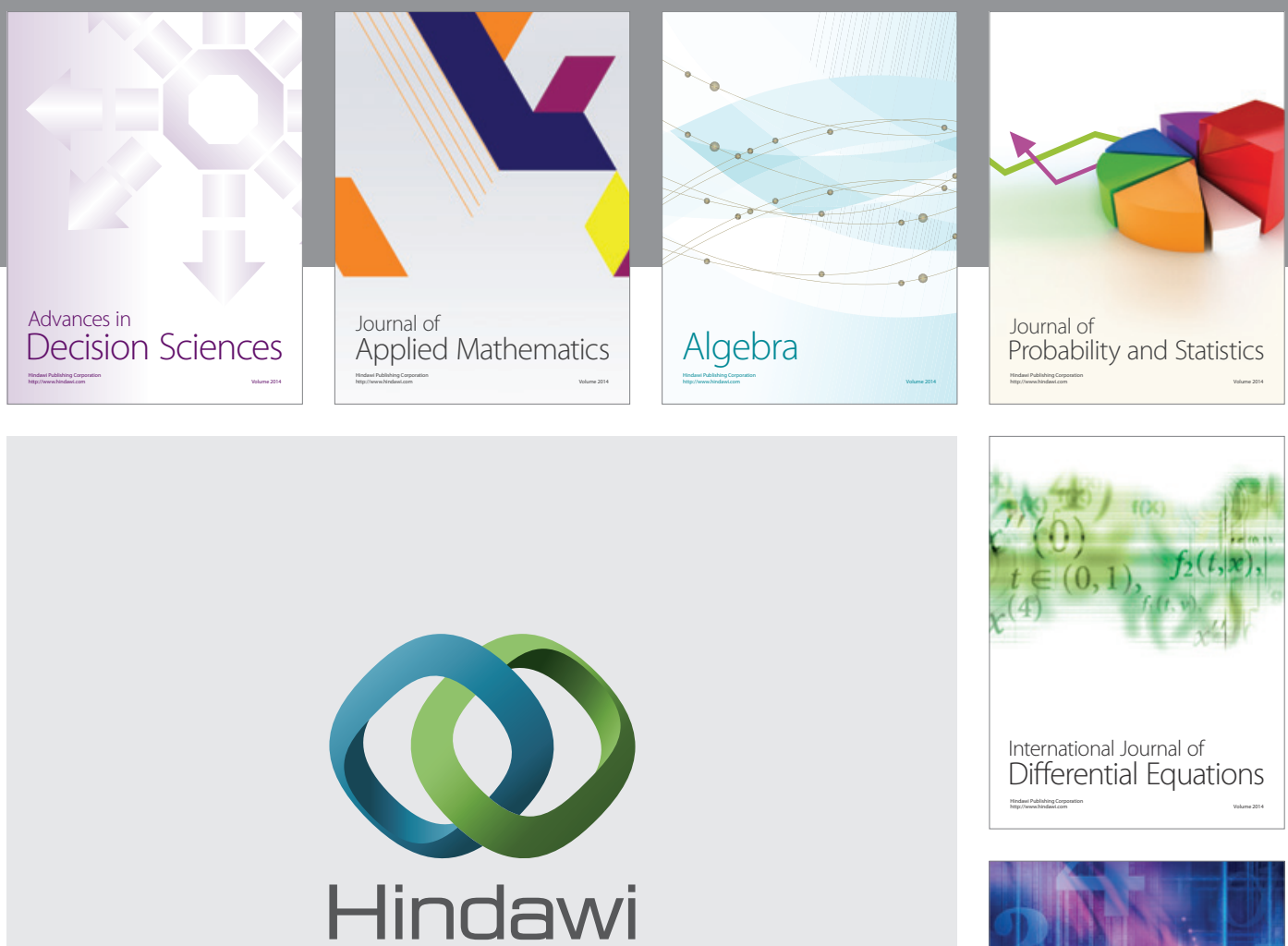

Submit your manuscripts at http://www.hindawi.com
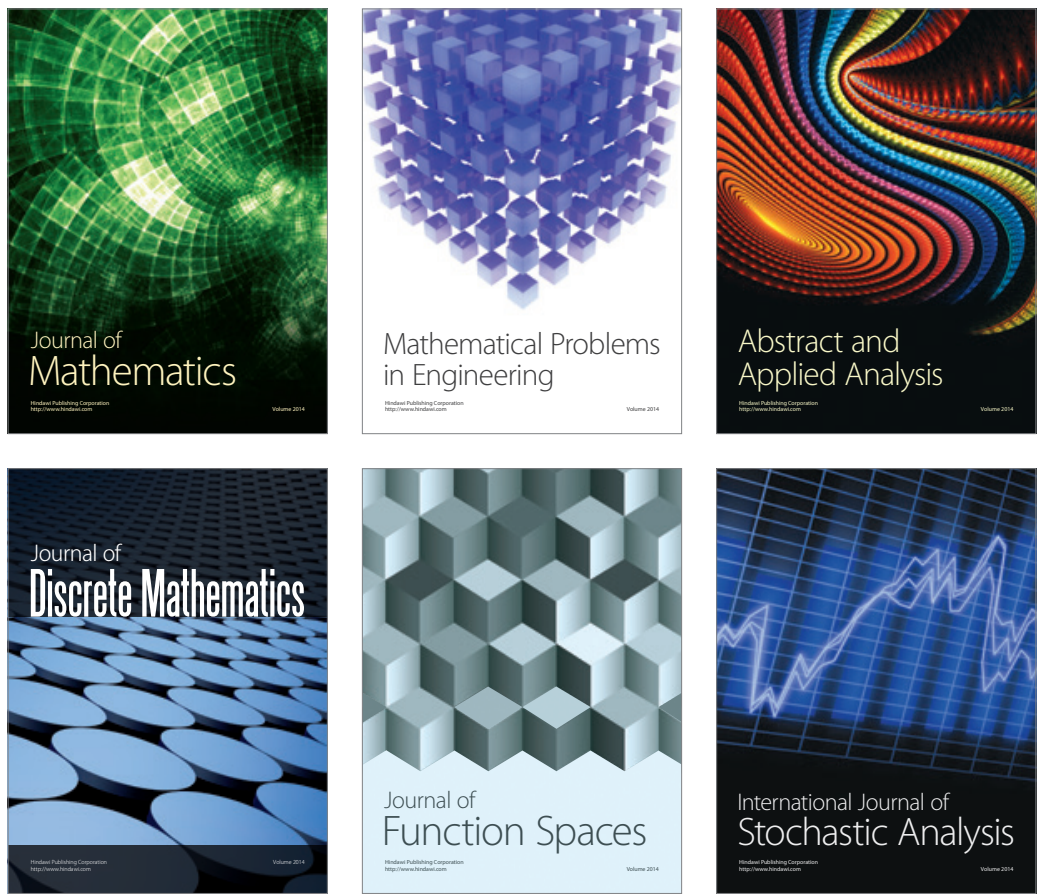

Journal of

Function Spaces

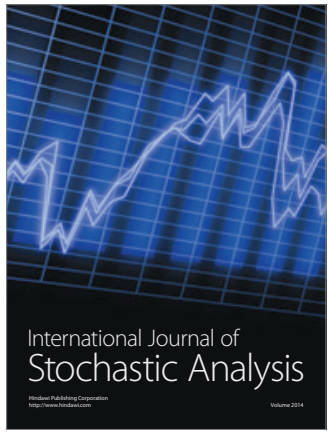

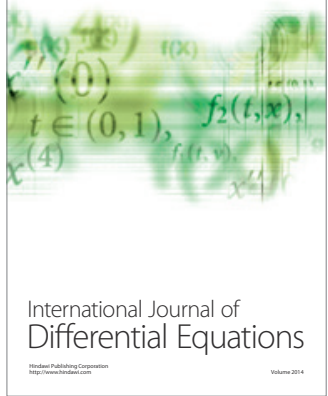
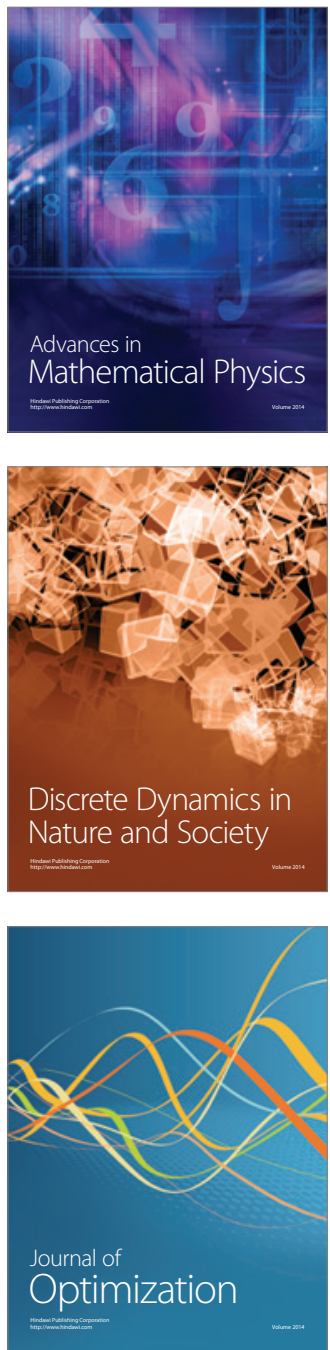